(C) Journal of Applied Mathematics \& Decision Sciences, 4(2), 175-182 (2000)

Reprints Available directly from the Editor. Printed in New Zealand.

\title{
Can we prevent the next epidemic? The elimination of childhood diseases by mass vaccination
}

M.G. ROBERTS

robertsm@agresearch.cri.nz

AgResearch, Wallaceville Animal Research Centre, P.O.Box 40063, Upper Hutt, New Zealand

\begin{abstract}
Recently in New Zealand there have been outbreaks of measles and pertussis every six and five years respectively. A model has been used to compare the dynamics of these diseases, and to determine the optimum ages at which children should be vaccinated against them. Whereas measles could be eliminated by giving the second vaccination at five years instead of eleven, it is difficult to devise a practical scheme that would eliminate pertussis. It is then necessary to consider vaccination schemes in the light of the age-structure of future epidemics as well as their timing.
\end{abstract}

\section{Introduction}

The childhood diseases that are controlled by vaccination programmes in New Zealand include measles, mumps, rubella and pertussis (commonly known as whooping cough) (Mansoor, 1999). Protection against the first three is via MMR vaccination at 15 months and eleven years, whereas four vaccinations against pertussis are given prior to 15 months. Despite these interventions epidemics of measles and pertussis still occur.

A quantity that is commonly used as an aid to understanding the population dynamics of an infectious disease is the basic reproduction ratio $\left(R_{0}\right)$. This is defined as the expected number of secondary cases that would arise from a single primary case introduced into a fully susceptible population (Diekmann et al., 1990; Anderson \& May, 1991; Roberts \& Heesterbeek, 1993). If $R_{0}<1$ the disease cannot persist in the population and will, over time, be eliminated. If $R_{0}>1$ the disease persists. Hence the objective of a disease elimination programme is to reduce the basic reproduction ratio below one. If a fraction $q$ of the population is immunised, then the basic reproduction ratio effectively becomes $R_{V}=(1-q) R_{0}$, and to eliminate the disease it is necessary to maintain an immune fraction $q>1-1 / R_{0}$, hence guaranteeing that $R_{V}<1$ (Edelstein-Keshet, 1988; Anderson \& May, 1991; Roberts \& Heesterbeek, 1993; McLean, 1994). Details of the community structure, especially patterns of contact between different age groups and at different times of the year, complicate this argument but do not fundamentally change it.

Until the introduction of measles vaccination in New Zealand in 1970 there was an epidemic of measles every two years. The effect of the vaccination programme was to increase the inter-epidemic period to six years, the last epidemic occurring in 1997. A mathematical model developed in 1996 successfully reproduced the 
historical pattern of epidemics and predicted the epidemic of 1997 (Mansoor et al., 1998; Tobias \& Roberts, 1998; Roberts \& Tobias, 2000). It was then shown that to reduce $R_{V}$ below one, and hence to prevent further epidemics, it is necessary to change the timing of the second vaccination from eleven years to six years or earlier (Tobias \& Roberts, 1998; Roberts \& Tobias, 2000). Similar models have been used to study the dynamics and control of measles in other countries, with similar results (e.g. Agur et al., 1993; Babad et al., 1995; Lévy-Bruhl et al., 1997; Gay et al., 1998).

Epidemics of pertussis occurred in New Zealand in 1971, 1974, 1978, 1982, 1986, 1991 and 1996 (Blakely 1999a). In this paper we report on an investigation into vaccination strategies against pertussis, and compare the results with those from the previous study for measles.

\section{The pertussis model}

We proceed by dividing a theoretical population that resembles New Zealand's into compartments, each of which has a specific disease status. Threshold conditions are then derived for the vaccination coverage that would be required to eliminate the disease. To demonstrate the method a population without age structure is considered. We then apply the method to a population where contact patterns depend on age. The model is similar to that previously used to model measles (Tobias \& Roberts, 1998; Roberts \& Tobias, 2000). The major difference is that, in contrast to measles, permanent immunity to pertussis is not conferred upon recovery or vaccination.

\subsection{The simple model}

Let the population of $N$ individuals be divided into $S$ susceptible to pertussis; $I$ infected and infectious; $R$ naturally immune; $P$ susceptible to infection but not disease (hence partially protected); $Q$ infected and infectious but not diseased; and $V$ vaccinated and immune. The flow rates between the compartments are shown in Figure 1. We assume a constant net birth rate $B$, and approximate early childhood vaccination by allocating a proportion $q$ of new-borns to the vaccinated compartment. Those vaccinated remain immune for an average period of $1 / \rho$ years, after which a proportion $r$ become susceptible to infection but not disease, and the remainder become susceptible to infection and disease. If a susceptible becomes infected, they remain infectious for an average period of $1 / \gamma$ years, then immune for $1 / \sigma$ years. At the end of the period of immunity a proportion $s$ become partially protected, the remainder becoming susceptible. If a member of the $P$ compartment is infected, he becomes infectious but not diseased for a mean time of $1 / \delta$ years, after which she joins the immune $(R)$ compartment. The force of infection from the $S$ compartment to the $I$ compartment is $\lambda=\beta I+\zeta Q$, and from the $P$ compartment to the $Q$ compartment $\kappa=\alpha I+\xi Q$. 


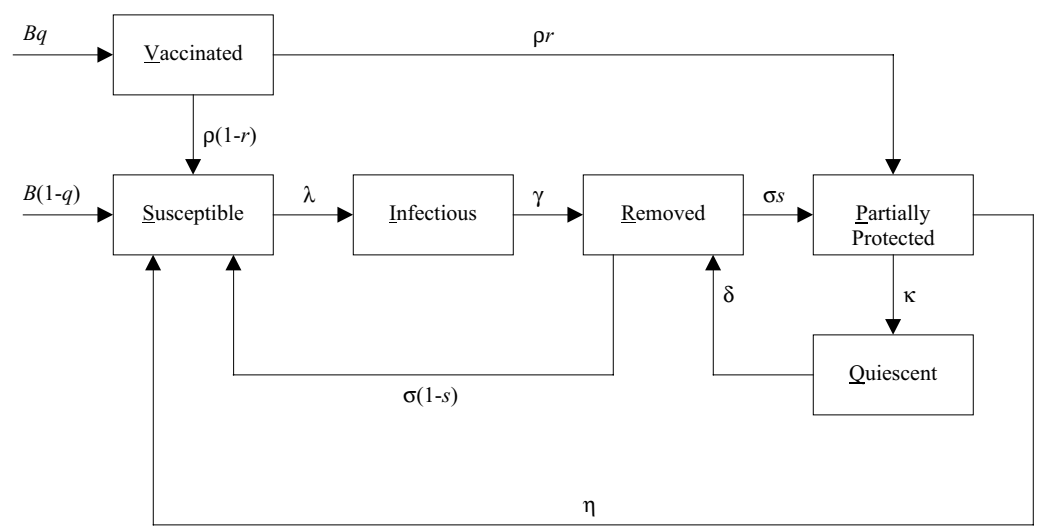

Figure 1. The structure of the model for the dynamics of pertussis. The model without age structure is shown, and for simplicity deaths (which affect all compartments equally) are not included on the diagram.

The equations describing the dynamics of the system are

$$
\begin{aligned}
\frac{d S}{d t} & =B(1-q)-(\beta I+\zeta Q+d) S+\sigma(1-s) R+\eta P+\rho(1-r) V \\
\frac{d I}{d t} & =(\beta I+\zeta Q) S-(\gamma+d) I \\
\frac{d R}{d t} & =\gamma I+\delta Q-(\sigma+d) R \\
\frac{d P}{d t} & =\sigma s R+\rho r V-(\alpha I+\xi Q) P-(\eta+d) P \\
\frac{d Q}{d t} & =(\alpha I+\xi Q) P-(\delta+d) Q \\
\frac{d V}{d t} & =B q-(\rho+d) V
\end{aligned}
$$

Choosing $N=B / d$, births and deaths balance and the population size is constant. The equations add to $d N / d t=0$, hence we can dispense with the need to model one variable explicitly. When no disease is present the steady state solutions are $I^{*}=Q^{*}=R^{*}=0$ and

$$
S^{*}=N-P^{*}-V^{*} \quad P^{*}=\frac{\rho r}{\eta+d} V^{*} \quad V^{*}=\frac{B q}{\rho+d}
$$

In the absence of vaccination $S^{*}=N, P^{*}=V^{*}=0$, and it is a trivial matter to show that the disease cannot maintain itself in the population when the basic reproduction number $R_{0}=\beta N /(\gamma+d)$ is less than one. Typical values of $R_{0}$ for pertussis in developed countries are in the range 16-18 (Anderson \& May, 1991). For more complex models $R_{0}$ can be obtained from the next generation matrix, 
which specifies the expected number of secondary infections in each compartment due to a single introduction of infection in one compartment in a susceptible population (Diekmann et al., 1990). For example, the next generation matrix derived from the above model in the presence of vaccination is

$$
M=\left(\begin{array}{ll}
\frac{\beta S^{*}}{\gamma+d} & \zeta S^{*} \\
\frac{\alpha P^{*}}{\gamma+d} & \frac{\xi P^{*}}{\delta+d}
\end{array}\right) .
$$

The entry $M_{11}$ represents the mean number of secondary infections in the $I$ compartment due to a primary infection in the $I$ compartment and an otherwise infection-free population. It is calculated by multiplying the transmission rate $\beta$, the number of susceptibles $S^{*}$, and the mean period of infectiousness $1 /(\gamma+d)$. Similarly $M_{12}, M_{21}$ and $M_{22}$ represent the numbers of secondary infections in the $I, Q$ and $Q$ compartments respectively due to primary infections in the $Q, I$ and $Q$ compartments respectively, in an otherwise infection-free population. A vaccination strategy will achieve elimination of the disease if $R_{V}=\mathcal{R}(M)<1$, where $\mathcal{R}$ signifies spectral radius. For the values of $R_{0}$ quoted above, vaccination coverage would have to maintain immunity in more than $93 \%$ of the population.

\subsection{The age-structured model}

A subdivision of the population into age groups allows for different inter-group contact rates, and provides a means by which strategies based on vaccinating children at defined ages may be modelled. We choose groups 0-9 to represent ages 0-3 months, 3-4 months, 4-5 months, 5-15 months, $1.25-3$ years, $3-5$ years, $5-11$ years, 11-17 years, $17-40$ years and $; 40$ years respectively. We assume that group 9 plays no part in the epidemic. Indexing compartments by age group number, our equations become:

$$
\begin{aligned}
\frac{d I_{0}}{d t} & =\lambda_{0} S_{0}-\left(\gamma+\mu_{0}\right) I_{0} \\
\frac{d R_{0}}{d t} & =\gamma I_{0}+\delta Q_{0}-\left(\sigma+\mu_{0}\right) R_{0} \\
\frac{d P_{0}}{d t} & =\sigma s R_{0}-\kappa_{0} P_{0}-\left(\eta+\mu_{0}\right) P_{0} \\
\frac{d Q_{0}}{d t} & =\kappa_{0} P_{0}-\left(\delta+\mu_{0}\right) Q_{0}
\end{aligned}
$$


and $V_{0}=0 ;$ and for $i=1 \ldots 8$

$$
\begin{aligned}
& \frac{d I_{i}}{d t}=\mu_{i-1} I_{i-1}+\lambda_{i} S_{i}-\left(\gamma+\mu_{i}\right) I_{i} \\
& \frac{d R_{i}}{d t}=\mu_{i-1} R_{i-1}+\gamma I_{i}+\delta Q_{i}-\left(\sigma+\mu_{i}\right) R_{i} \\
& \frac{d P_{i}}{d t}=\mu_{i-1} P_{i-1}+\sigma s R_{i}+\rho r V_{i}-\kappa_{i} P_{i}-\left(\eta+\mu_{i}\right) P_{i} \\
& \frac{d Q_{i}}{d t}=\mu_{i-1} Q_{i-1}+\kappa_{i} P_{i}-\left(\delta+\mu_{i}\right) Q_{i} \\
& \frac{d V_{i}}{d t}=\mu_{i-1} V_{i-1}+q_{i} \mu_{i-1} S_{i-1}-\left(\rho+\mu_{i}\right) V_{i}
\end{aligned}
$$

where

$$
\lambda_{i}=\beta \sum_{j=0}^{8} C_{i j} I_{j}+\zeta \sum_{j=0}^{8} C_{i j} Q_{j}
$$

and

$$
\kappa_{i}=\alpha \sum_{j=0}^{8} C_{i j} I_{j}+\xi \sum_{j=0}^{8} C_{i j} Q_{j}
$$

are the forces of infection on the susceptible and partially protected compartments respectively, and the terms $C_{i j}$ specify relative rates of contact between different age groups (see below).

The transition rates between age groups $\mu_{i}$ are the reciprocals of the age group width, assuming the death rate is negligible below 40 years of age. Hence the size of each age group is constant and equal to the group width multiplied by the birth rate, which was set at $B=57435$ person yr. ${ }^{-1}$ to equal that of New Zealand in 1994 (Statistics New Zealand, 1996). The proportion of the population that is successfully vaccinated on entering group $i$ is $q_{i}$. Due to the uncertainty of the timing of interventions in the first few weeks of life, the first two vaccinations were approximated by one at 3 months (Blakely et al., 1999b), with subsequent vaccinations at 5 months and 15 months.

Grenfell \& Anderson (1989) used a latent period of 7 days and an infectious period of 22 days in their model of pertussis, whereas Anderson \& May (1991) quote an incubation period of $6-10$ days, latent period of 21-23 days and an infectious period of $7-10$ days. Hence we set $\gamma=\delta=12.6 \mathrm{yr}^{-1}$ Other flow rates estimated from the literature were $\rho=\sigma=0.1 \mathrm{yr}^{-1}$ and $\eta=0$ (see e.g. Grenfell \& Anderson, 1989; Hethcote, 1997). The proportions remaining partially protected following loss of immunity were assumed to be $r=s=0.9$, and in the absence of better information we set $\alpha, \beta, \xi$ and $\zeta$ all equal to $4.06 \times 10^{-5}$ person $^{-1}$ yr. $^{-1}$, to achieve a suitable value of $R_{0}$ (see below).

The contact rates $C_{i j}$ are based on those used for measles (Roberts \& Tobias, 2000). We define activity levels $a_{1}=1$ for infants (age groups 0-3), $a_{2}=3$ for 
pre-schoolers (groups 4-5), $a_{3}=6$ for primary school children (group 6), and $a_{4}=$ $a_{5}=3$ for secondary school children and adults (groups 7\&8) respectively. Within group contact rates are then $C_{i i}=a_{k(i)}$, and inter-group contact rates are $C_{i j}=$ $\varepsilon \sqrt{a_{k(i)} a_{k(j)}}$ if $k(i) \neq k(j)$, where $k(i)=1$ if $i \in[0,3], k(i)=2$ if $i \in[4,5]$, and $k(i)=i-3$ if $i \in[6,8]$. This process is equivalent to specifying a WAIFW ("who acquires infection from whom") matrix (Anderson \& May, 1991; Hethcote, 1996). By comparison with the measles model we set $\varepsilon=0.4$ (Roberts \& Tobias, 2000).

The next generation matrix in the absence of vaccination is defined by

$$
\left(M_{0}\right)_{i+1, j+1}=\frac{\beta C_{i j} N_{i}+\epsilon_{i-1, j} \mu_{j}}{\mu_{i}+\gamma}
$$

where $\epsilon_{i-1, j}=1$ if $i-1=j$ and zero otherwise. We then have $R_{0}=\mathcal{R}\left(M_{0}\right)$. Using the parameter values above we obtain $R_{0}=15.8$.

The steady state under vaccination with no disease solves

$$
V_{i}^{*}=\frac{q_{i} \mu_{i-1} S_{i-1}^{*}+\mu_{i-1} V_{i-1}^{*}}{\rho+\mu_{i}} \quad P_{i}^{*}=\frac{\mu_{i-1} P_{i-1}^{*}+\rho r V_{i}^{*}}{\eta+\mu_{i}}
$$

and $S_{i}^{*}=N_{i}-P_{i}^{*}-V_{i}^{*}$ for $i=0 \ldots 8$, with $S_{0}^{*}=N_{0}$ and $P_{0}^{*}=V_{0}^{*}=0$. The next-generation matrix is then

$$
M_{V}=\left(\begin{array}{ll}
M_{1} & M_{2} \\
M_{3} & M_{4}
\end{array}\right)
$$

where $M_{1}$ is equal to $M_{0}$ with $N_{i}$ replaced by $S_{i}^{*} ; M_{2}$ is equal to $M_{0}$ with $N_{i}$ replaced by $S_{i}^{*}$ and $\beta$ replaced by $\zeta ; M_{3}$ is equal to $M_{0}$ with $N_{i}$ replaced by $P_{i}^{*}, \beta$ replaced by $\alpha$ and $\gamma$ replaced by $\delta$; and $M_{4}$ is equal to $M_{0}$ with $N_{i}$ replaced by $P_{i}^{*}, \beta$ replaced by $\xi$ and $\gamma$ replaced by $\delta$. The basic reproduction ratio under vaccination is then $R_{V}=\mathcal{R}\left(M_{V}\right)$. Estimates of current vaccine coverage in New Zealand are $80-90 \%$ by 3 months and at 5 months, and $80 \%$ at 15 months. Efficacy is estimated at 50$80 \%$ by 3 months and $80-95 \%$ at 5 and 15 months. Hence $q_{1}=0.5525, q_{3}=0.74375$, $q_{4}=0.7, q_{i}=0$ otherwise; leading to $R_{V}=13.33$. Further epidemics of pertussis are therefore inevitable with the present strategy.

\section{Optimising vaccination strategies}

The model described above is more complex than that used by Roberts \& Tobias (2000) to describe the epidemiology of measles in New Zealand. In fact, the measles model may be derived from it by deleting the $P$ and $Q$ compartments, and changing parameter values. In particular, immunity to measles may be regarded as life-long whether it is acquired through vaccination or infection, hence $\rho=\sigma=0$. For measles we have $R_{0}=12.8$, which is reduced by the current vaccination programme to $R_{V}=2.85$. The current programme based on vaccinations at 15 months and eleven years, leaves an interval between treatments sufficient for the number not effectively vaccinated to accumulate to a critical level. Hence to eradicate measles 
it is necessary to bring forward the timing of the second vaccination, school entry at five years would provide a suitable opportunity.

The results obtained for pertussis vaccination showed that eradication is a long way from being achieved. The fundamental difference between the two diseases is the lack of permanent immunity conferred by infection or vaccination against pertussis. It was determined that if a pertussis vaccine were to confer life-long immunity, the current vaccination schedule would achieve $R_{V}=0.64$. These results are similar to those obtained from a similar study in the USA (Hethcote, 1997). However, childhood vaccination against pertussis does reduce the number of cases in early years when the effects of the disease are more severe, and is therefore beneficial in itself.

The pattern of repeated epidemics is due to seasonal forcing of transmission arising from closer contact between children during the school year (Grenfell \& Bolker, 1994). Numerical solution of the measles model confirmed this, and established a relationship between $R_{V}$ for the non-forced model and the inter-epidemic period of the forced model. Although the values of $R_{V}$ are not equal for the two models, a lower value of $R_{V}$ calculated from the non-forced model would lead to less frequent epidemics in the seasonally forced model, and $R_{V} \ll 1$ implies elimination of the disease. It has been found in the UK that regular epidemics of measles, correlated between major cities, have given way to irregular uncorrelated outbreaks postvaccination; whereas pertussis vaccination strategies have synchonised a previously uncorrelated epidemic pattern (Rohani et al., 1999). It is argued that the longer infectious period of pertussis makes the dynamics less sensitive to seasonal forcing, and concluded that pertussis outbreaks can be explained by simple deterministic arguments. The success of our previous investigation into measles provides hope that this may be the case in New Zealand, and that analytical tools may prove valuable in optimising vaccination strategies against the common childhood diseases.

\section{Acknowledgments}

The author would like to thank Martin Tobias and Osman Mansoor of the New Zealand Ministry of Health for introducing him to this topic. Some of the material was prepared under contract for the ministry, but the conclusions do not necessarily represent the ministry's views. Herbert Hethcote, Angela McLean and Valerie Isham are also acknowledged for helpful discussions, and Glenn Fulford provided comments on an earlier draft that improved the presentation.

\section{References}

1. Agur Z, Cojocaru L, Mazor G, Anderson RM, Danon YL. 1993. Pulse mass measles vaccination across age cohorts. Proceedings of the National Academy of Sciences USA 90:1169811702 .

2. Anderson RM, May RM. 1991. Infectious diseases of humans: dynamics and control. Oxford: Oxford University Press. 
3. Babad HR, Nokes DJ, Gay NJ, Miller E, Morgan-Capner P, Anderson RM. 1995. Predicting the impact of measles vaccination in England and Wales: model validation and analysis of policy options. Epidemiology and Infection 114:319-344.

4. Blakely TA, Mansoor O, Baker M. 1999a. The 1996 pertussis epidemic in New Zealand: descriptive epidemiology. New Zealand Medical Journal 112:30-33.

5. Blakely TA, Mansoor O, Baker M. 1999b. The 1996 pertussis epidemic in New Zealand: vaccine effectiveness. New Zealand Medical Journal 112:118-120.

6. Diekmann O, Heesterbeek JAP, Metz JAJ. 1990. On the definition and computation of the basic reproduction ratio $R_{0}$ in models for infectious diseases in heterogeneous populations. Journal of Mathematical Biology 28:365-382.

7. Edelstein-Keshet L. 1988. Mathematical models in biology. New York: Random House.

8. Gay NJ, Pelletier L, Duclos P. 1998. Modelling the incidence of measles in Canada: an assessment of the options for vaccination policy. Vaccine 16:794-801.

9. Grenfell BT, Anderson RM. 1989. Pertussis in England and Wales: an investigation of transmission dynamics and control by mass vaccination. Proceedings of the Royal Society of London B 236:213-252.

10. Grenfell BT, Bolker BM. 1994. Population dynamics of measles. In Parasitic and Infectious Diseases, edited by Scott ME, Smith G. New York, Academic Press:219-233.

11. Hethcote HW. 1996. Modelling heterogeneous mixing in infectious disease dynamics. In Models for infectious human diseases: their structure and relation to data, edited by Isham VS, Medley GF. Cambridge, Cambridge University Press:215-238.

12. Hethcote HW. 1997. An age-structured model for pertussis transmission. Mathematical Biosciences 145:89-136.

13. Lévy-Bruhl D, Maccario J, Richardson S, Guérin N. 1997. Modélisation de la rougeole en France et conséquences pour l'âge d'administration de la seconde vaccination rougeoleoreillons-rubéole. Bulletin Epidémiologique Hebdomadaire 29:133-135.

14. Mansoor O, Blakely A, Baker M, Tobias M, Bloomfield A. 1998. A measles epidemic controlled by immunisation. New Zealand Medical Journal 111:467-471.

15. Mansoor O, Reid S. 1999. The future of the immunisation schedule: recommendations of a workshop. New Zealand Medical Journal 112:52-55.

16. McLean AR. 1994. Control of microparasites through vaccination. In Parasitic and Infectious Diseases, edited by Scott ME, Smith G. New York, Academic Press:129-140.

17. Roberts MG, Heesterbeek JAP. 1993. Bluff your way in epidemic models. Trends in Microbiology 1:343-348.

18. Roberts MG, Tobias MI. 2000. Predicting and preventing measles epidemics in New Zealand: Application of a mathematical model. Epidemiology and Infection 124:279-287.

19. Rohani P, Earn DJD, Grenfell BT. 1999. Opposite patterns of synchrony in sympatric disease metapopulations. Science 286:968-971.

20. Statistics New Zealand. 1996. New Zealand Official Yearbook. Wellington: Statistics New Zealand.

21. Tobias MI, Roberts MG. 1998. Modelling measles. Predicting and preventing measles epidemics in New Zealand: Application of a mathematical model. Wellington: Ministry of Health. 


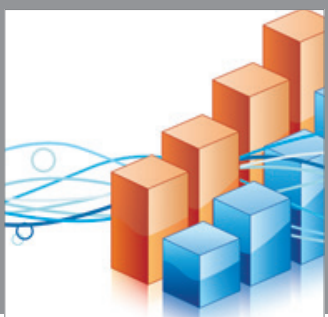

Advances in

Operations Research

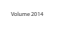

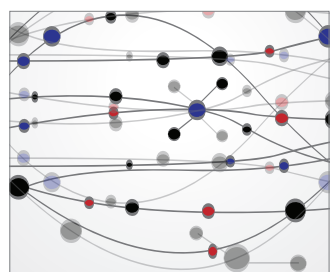

\section{The Scientific} World Journal
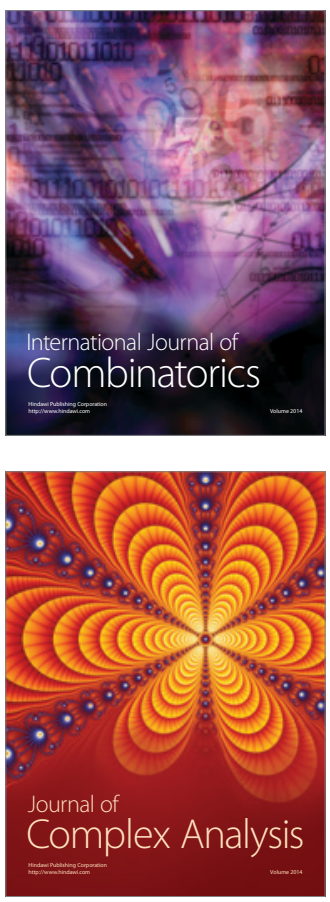

International Journal of

Mathematics and

Mathematical

Sciences
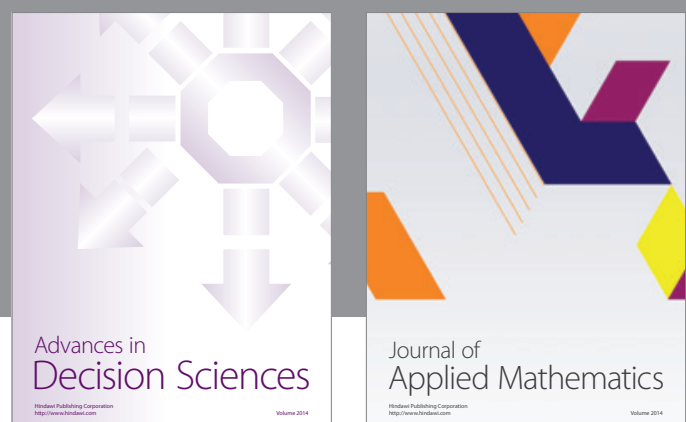

Journal of

Applied Mathematics
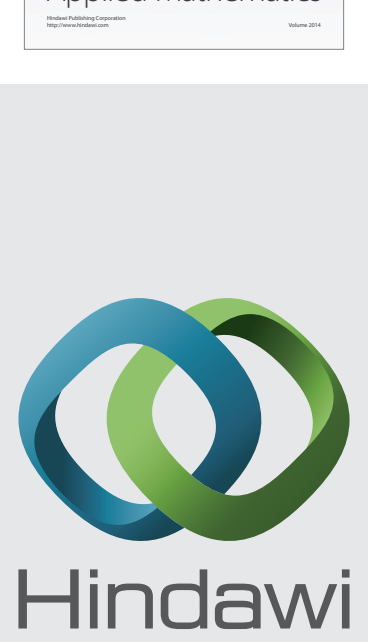

Submit your manuscripts at http://www.hindawi.com
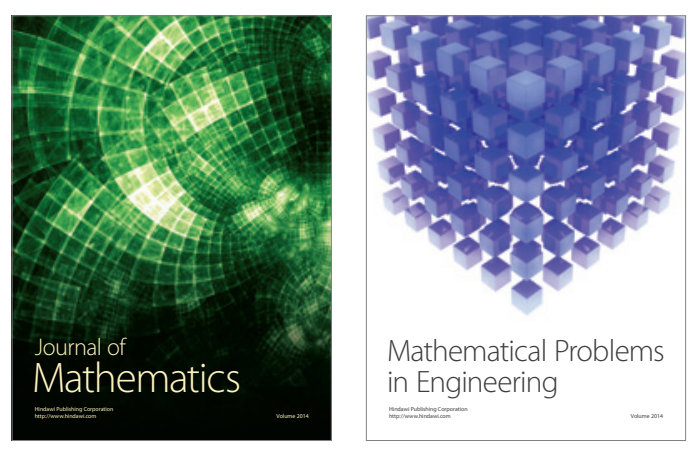

Mathematical Problems in Engineering
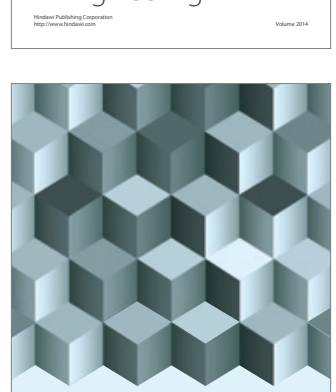

Journal of

Function Spaces
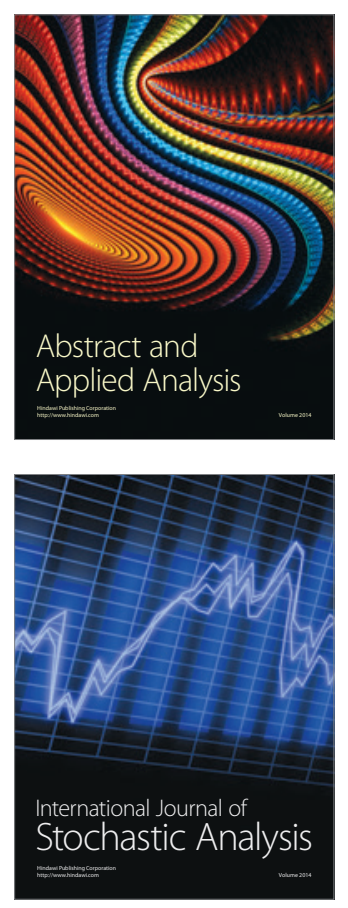

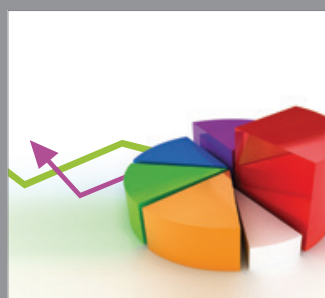

ournal of

Probability and Statistics

Promensencen
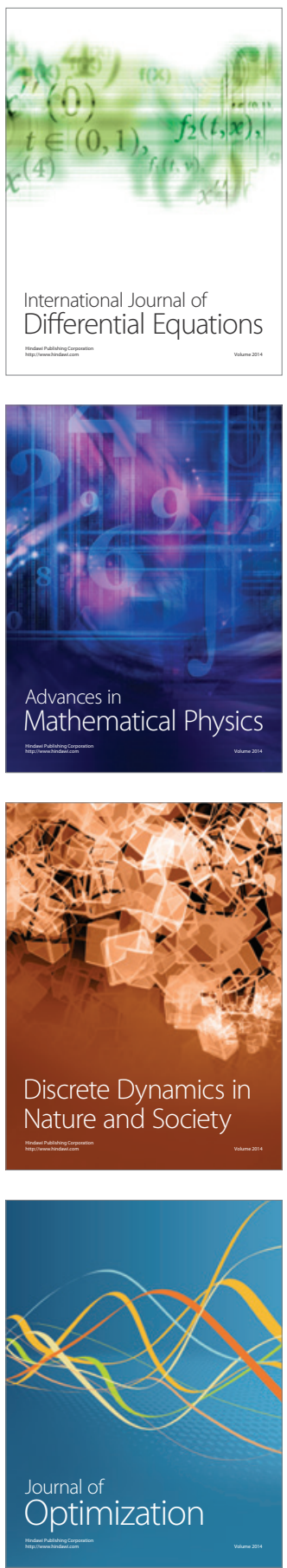